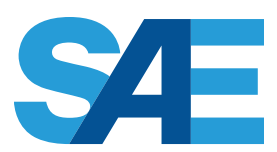

INTERNATIONAL.

\title{
Spray Modeling for Outwardly-Opening Hollow-Cone Injector
}

\section{Jaeheon Sim}

KAUST

\section{Jihad Badra}

Saudi Aramco

\section{Ahmed Elwardany and Hong Im \\ KAUST}

CITATION: Sim, J., Badra, J., Elwardany, A., and Im, H., "Spray Modeling for Outwardly-Opening Hollow-Cone Injector," SAE Technical Paper 2016-01-0844, 2016, doi:10.4271/2016-01-0844.

Copyright (C) 2016 SAE International

\begin{abstract}
The outwardly-opening piezoelectric injector is gaining popularity as a high efficient spray injector due to its precise control of the spray. However, few modeling studies have been reported on these promising injectors. Furthermore, traditional linear instability sheet atomization (LISA) model was originally developed for pressure swirl hollow-cone injectors with moderate spray angle and toroidal ligament breakups. Therefore, it is not appropriate for the outwardlyopening injectors having wide spray angles and string-like film structures. In this study, a new spray injection modeling was proposed for outwardly-opening hollow-cone injector. The injection velocities are computed from the given mass flow rate and injection pressure instead of ambiguous annular nozzle geometry. The modified Kelvin-Helmholtz and Rayleigh-Taylor (KH-RT) breakup model is used with adjusted initial Sauter mean diameter (SMD) for modeling breakup of string-like structure. Spray injection was modeled using a Lagrangian discrete parcel method within the framework of commercial CFD software CONVERGE, and the new model was implemented through the user-defined functions. A Siemens outwardly-opening hollow-cone spray injector was characterized and validated with existing experimental data at the injection pressure of 100 bar. It was found that the collision modeling becomes important in the current injector because of dense spray near nozzle. The injection distribution model showed insignificant effects on spray due to small initial droplets. It was demonstrated that the new model can predict the liquid penetration length and local SMD with improved accuracy for the injector under study.
\end{abstract}

\section{Introduction}

Understanding spray atomization of liquid fuels is crucial for the development of high efficiency and low emission engines because the large surface area of a finely atomized spray enhances fuel evaporation and hence mixing and combustion $[1,2]$. Recently, the spray atomization drew increased interests because gasoline direct injection (GDI) and gasoline compression ignition (GCI) engines have been considered as alternatives to the traditional spark ignition and Diesel engines. Diesel engine has been known as highly efficient lean burn compression ignition with no-throttling losses but requires complicated and expensive fuel injection and after-treatment system, whereas, gasoline spark ignition engine is relatively simple but suffers from reduced thermal efficiency. GCI engines in general operates at low temperature combustion (LTC) conditions that have been gaining increasing attention over the last decade due to its potential of achieving diesel-like thermal efficiencies with significantly reduced engine-out nitrogen oxides (NOx) and soot emissions. Recent studies $[\underline{3}, \underline{4}, \underline{5}]$ reported that GCI combustion occurs as a series of autoignition events with minor contributions from the flame fronts. As such, successful GCI operation is usually achieved by controlling the autoignition timing by manipulating equivalence ratio stratification levels within the cylinder through late injection in the compression stroke. Therefore, an appropriate spray modeling of the recent high efficiency injector is required for the precise control of direct fuel injection without throttle losses. This is crucial in designing engines with high thermodynamic efficiencies and low emissions. 
Pressure-swirling and inwardly-opening hollow-cone spray injector has been commonly utilized in GDI and GCI engines due to its efficient atomization with relatively wide spray angle and fine atomization $[\underline{6}, \underline{7}, \underline{8}]$. Recently, the outwardly-opening piezoelectric injector is gaining popularity as a high efficient hollow-cone spray injector due to its precise control of the spray by accurate piezoelectric actuator [9]. However, few modeling studies are available on these promising high efficient injectors.

In spray modeling, a Lagrangian discrete parcel method is widely used as a computationally efficient representation of liquid droplets and their interaction with gas phase flow fields. One of the key aspects in the Lagrangian spray model is how to describe the spray break-up process. It has been known that the typical KelvinHelmholtz and Rayleigh-Taylor (KH-RT) breakup model is unable to describe the hollow-cone spray because the liquid is injected with circular-shaped liquid sheet instead of a cylindrical blob. As such, Schmidt et al. [7] proposed the linear instability sheet atomization (LISA) model by assuming toroidal ligament breakup for pressureswirl hollow-cone spray injectors, where the Taylor analogy breakup (TAB) was used as a secondary breakup model after primary LISA breakup. The LISA-TAB model was further applied to the outwardlyopening injector by assuming slug flow, although linear instability theory was unable to model the actual breakups of expanding 3-D liquid sheets []. Das [10] simulated outwardly-opening hollow-cone spray using the TAB model by specifying the gap height as the initial thickness of the liquid blob, but the liquid penetration measurement showed large deviations from experiment except initial injection period. Pischke et al. [11] proposed a combined LISA-KH-TAB model by adding KH breakup as a secondary breakup for high Weber number conditions because TAB model produces unreasonably fine droplets at that condition.

The LISA model has been the most common approach for simulating the liquid sheet breakup of hollow-cone spray, and shows good agreement on the traditional pressure-swirl injector having toroidal ligaments by transverse swirling motions. However, recent experimental studies reported that some modern outwardly-opening piezoelectric injector produces hollow-cone spray with string-like film structures instead of toroidal ligaments from the nozzle exit [12]. The mechanism of these string-like structures has not yet been clearly understood, and no appropriate models to predict such a string formation and accurate droplet sizes exist in the literature.

Collision modeling between droplets is another key component in the spray simulation because it has huge influence on the drop sizes, and thus overall spray characteristics. O'Rourke collision model [13] has been widely used to calculate collision probability and coalescence rate in Lagrangian discrete parcel method although the model accounts for only two collision regimes for simplicity; coalescence and stretching separation. Post and Abraham [14] suggested a new collision outcome model including experimentally-observed collision behavior of hydrocarbon drops under four different collision regimes with both bouncing and reflexive separation, thereby covering all the regimes when the collision Weber number is not too high. It is known that Post's outcome model shows better accuracy in dense Diesel spray at high chamber pressure [14]. Munnannur et al. [15] developed more advanced O'Rourke-type collision model by including the known four collision regimes and adding fragmentations in stretching and reflective mechanism. However, the O'Rourke model is still a widely used standard model in spray modeling. Most previous studies on hollow-cone spray used the original O'Rourke model. Pischke et al.[11] implemented Munnannur's outcome model without any consideration of satellite droplets in the modeling of hollow-cone spray, but the difference of spray characteristics between models was not reported.

In the present study, the outwardly-opening hollow-cone injector was modeled by CONVERGE [16], a commercial CFD software specified for the engine simulation, using user-defined function (UDF) features. A new spray injection model for outwardly-opening hollow-cone injector is proposed. The injection velocities are computed from the given mass flow rate and injection pressure instead of ambiguous annular nozzle geometry. To model breakup of string-like film structure, the modified KH-RT breakup model is used with adjusted initial SMD. Various collision models, and injection distribution models were tested and the results were compared to the experimental data to identify the optimal model description for the specific injector configuration.

\section{Spray Modeling}

The numerical modeling of a hollow-cone spray in a constant volume chamber is conducted by CONVERGE [16] using user-defined functions. For turbulence modeling, the RANS-based renormalization group (RNG) $\mathrm{k}-\varepsilon$ model is utilized, and a Lagrangian discrete parcel method is used for spray modeling by introducing parcels (groups of droplets) of liquid into the gas phase computational domain with the calculated injection velocities and initial SMD distributions. As a breakup algorithm of liquid spray, the modified Kelvin-Helmholtz and Rayleigh-Taylor (KH-RT) model is mainly used without an ad hoc breakup length [17].

\section{Spray Injection Velocity}

How to inject liquid spray is the most important part in the hollowcone spray modeling. For a solid cone spray, the injection itself is straightforward because the liquid is injected from the fixed circular nozzle exit having relatively large constant nozzle diameter (bigger than $0.2 \mathrm{~mm}$ ). Injection velocities can be computed from mass flow rate and effective nozzle area although cavitation effect adds more difficulties. However, there exist an air-core and transverse injection velocities in a pressure-swirl inwardly-opening hollow-cone injector. For outwardly-opening injector, the nozzle exit looks like an annular gap, but the area and cut-plane change by needle lift. Schmidt et al. [7] proposed LISA breakup model with an injection velocity formula by accounting an air-core formation based on mass conservation and Bernoulli equation for a pressure-swirl inwardly-opening injector. Senecal et al. [] ] applied the LISA model to outwardly-opening injector by computing injection velocity from mass flow rate and the constant annular gap size like solid cone. However, the direction of annular nozzle exit as well as annular gap area change with injector needle motions. Furthermore, the nozzle exit flow from the very small annular gap size, less than $25 \mu \mathrm{m}$, makes the implementation of previous approaches more difficult. 
The details of nozzle flow and the inside structure of the outwardlyopening injector is mostly unknown. However, the steady injection velocity can be estimated by the Bernoulli equation and the nozzle's discharge coefficient $\left(C_{D}\right)$. This approach is more reasonable choice than Senecal's slug flow assumption because the annular gap is very small and nozzle exit direction and area change in time. The steady injection velocity $\left(V_{i n j}\right)$ can be computed from the liquid density $\left(\rho_{l}\right)$ and pressure difference $(\Delta p)$ between the injection pressure $\left(p_{i n j}\right)$ and chamber pressure $\left(p_{c}\right)$ :

$$
V_{i n j}=C_{D} \sqrt{\frac{2\left(p_{i n j}-p_{c}\right)}{\rho_{l}}}
$$

In the original LISA breakup model for pressure-swirl injectors, a velocity coefficient $\left(k_{v}\right)$ is used instead of discharge coefficient $\left(C_{D}\right)$ in Eq. (1), and a correlation for the coefficient has been suggested [ $\underline{8}$ ]. Later, Schmidt et al.[7] suggested 0.7 as a velocity coefficient from a typical value ( 0.78 ) of the discharge coefficient of a single nozzle with sharp inlet corners by reducing the value by $10 \%$ for extra momentum losses and accounting for limitations of air core. In the present study, the discharge coefficient of 0.75 was obtained from the experimental results, and is similar to that of single nozzle with sharp inlet corner.

\section{Spray Liquid Sheet Thickness and Initial SMD}

The initial liquid sheet thickness $\left(t_{0}\right)$ is computed from the injection velocity and mass conservation equation unlike solid cone or Senecal's approach, where the liquid sheet thickness is the known value and is same to the nozzle diameter or annular gap. In the present study, the sheet thickness is different from the annular gap size because the injection velocity and liquid sheet thickness are estimated at a little distance from the nozzle exit after the nozzle flow is fully developed. When the mass flow rate $(\dot{m})$ is known, the initial sheet thickness of the liquid film $\left(t_{0}\right)$ can be obtained from the injection velocity $\left(V_{i n j}\right)$ and nozzle diameter $\left(d_{n}\right)$ by

$$
\dot{m}=\pi \rho_{l} V_{i n j} t_{0}\left(d_{n}-t_{0}\right)
$$

The sheet thickness is defined on the plane perpendicular to the injection velocity unlike Schmidt's approach, where it is defined as perpendicular to the axial direction of nozzle. The calculated liquid sheet thickness is a starting point in our spray modeling regardless of breakup or collision models. In the present study, modified KH-RT breakup models are implemented by assuming string-like film structure of the outwardly-opening hollow-cone injector because LISA model represents toroidal ligaments breakup in the pressure swirl injector. For the present spray modeling, the initial SMD is the most ambiguous parameter. The initial liquid sheet thickness $\left(t_{0}\right)$ of Eq. (2) can be considered as an initial SMD. However, it does not comply with string-like structures and the liquid jet stability analysis of the Kelvin-Helmholtz instability. Instead, the spray is considered many small solid cone injections along a circular liquid sheet in favor of the experimentally observed string-like structure of the outwardlyopening hollow-cone spray injection. Assuming that the cylindrical liquid blobs are attached each other, the initial SMD $\left(D_{0}\right)$ for the KHRT breakup model is computed by

$$
\pi t_{0}\left(d_{n}-t_{0}\right)=\frac{\pi}{4} D_{0}^{2} \frac{\pi\left(d_{n}-t_{0}\right)}{D_{0}}
$$

By simplifying the equation, the initial SMD is computed by

$$
D_{0}=\frac{4}{\pi} t_{0}
$$

The equation (4) is used as an initial SMD through this study. This value is not a constant, but a function of liquid film thickness, which is computed from the given mass flow rate and computed injection velocity. The similar idea was tested in our previous study [18] to optimize spray models for GCI engines based on penetration length, but the sheet thickness was measured perpendicular to axial direction and assumed constant unlike this study. Previous experimental researches $[11,12]$ show string-like film structures from the nozzle exit clearly, and this supports our approach, where the strings are formed from imaginary circular liquid film. In the results section, the local SMD values are bigger than maximum annual gab size, and this

\begin{tabular}{|c|c|c|c|c|c|}
\hline & Senecal et al. (1999) [8] & Das (2008) [10] & Pischke et al. (2010) [11] & $\begin{array}{c}\text { Built-in model in Converge } \\
{[16] \text { and Fluent }}\end{array}$ & Present study \\
\hline Known values & \multicolumn{2}{|c|}{$t_{0}, \quad \dot{m}$} & $t_{0}$ profile, $\boldsymbol{P}_{i n j}$ & \multirow{4}{*}{$\begin{array}{l}\text { There is no intrinsic option } \\
\text { for outwardly-opening } \\
\text { injector. Therefore, the } \\
\text { approach by Senecal et al. } \\
\text { (1999) should be used for } \\
\text { spray injection. }\end{array}$} & $\dot{m}, P_{i n j}$ \\
\hline Injection velocity $\left(V_{\text {inj }}\right)$ & $V_{i n j}=$ & & $\begin{array}{l}\text { Bernoulli Eq. (from } \\
\text { pressure difference) }\end{array}$ & & $\begin{array}{l}\text { Bernoulli Eq. (from } \\
\text { pressure difference) }\end{array}$ \\
\hline Discharge coefficient $\left(C_{D}\right)$ & & & 0.756 (from exp.) & & 0.75 (from exp.) \\
\hline $\begin{array}{l}\text { Initial liquid film thickness } \\
\qquad\left(\mathrm{t}_{0}\right)\end{array}$ & \multicolumn{3}{|c|}{ Gap height (assumed known) } & & $\begin{array}{l}\text { Calculated from mass } \\
\text { conservation }\end{array}$ \\
\hline Ligament diameter $\left(\mathrm{d}_{\mathrm{L}}\right)$ & \multicolumn{3}{|c|}{$\begin{array}{l}\qquad d_{L}=\sqrt{\frac{16\left(t_{0} / 2\right)}{K_{s}}} \text { for short wave (From original LISA theory) } \\
* \mathrm{~K}_{\mathrm{S}} \text { is the wave number corresponding to the maximum growth rate of the wave }\end{array}$} & $d_{L}=C_{\text {lisa }} \frac{2 \pi}{K_{s}}$ & \multirow{2}{*}{$\begin{array}{c}D_{0}=(4 / \pi) t_{0} \\
\text { Calculated from mass } \\
\text { conservation } \\
\text { (String fromation from } \\
\text { liquid film) } \\
\end{array}$} \\
\hline Initial $\mathrm{SMD}\left(\mathrm{d}_{\mathrm{D}}\right.$ or $\left.\mathrm{D}_{0}\right)$ & \multicolumn{4}{|c|}{$\begin{array}{c}d_{D}=1.88 d_{L}(1+30 h)^{1 / 6} \\
O h=\mu_{l} /\left(\rho_{l} \sigma d_{L}\right)^{1 / 2} \text { (From original LISA theory) } \\
\text { (Ohnesorge number) }\end{array}$} & \\
\hline Primary breakup & LISA & & LISA & LISA & \multirow{2}{*}{$\begin{array}{l}\text { Modified KH-RT w/o } \\
\text { breakup length }\end{array}$} \\
\hline Secondary breakup & $\mathrm{TAB}$ & $\mathrm{TAB}$ & TAB, KH & $\mathrm{TAB}$ & \\
\hline
\end{tabular}
indicates that our approach on initial SMD can be a more reasonable choice than typical annular gap size.

Table 1. Summary of various spray modeling for outwardly-opening hollow-cone injector. 
The constant injection droplet size, Chi-squared distribution, and Rosin-Rammler injection size distribution with a parameter of 3.5 are tested and compared for the best spray injection modeling.

Furthermore, the dynamic drag model was implemented by determining the droplet drag coefficient dynamically, accounting for variations in the drop shape through a drop distortion parameter for accurate spray modeling. The effect of the turbulent flow on spray drops is modeled using O'Rourke's turbulent dispersion model by accounting a fluctuating velocities [13]. For collision model, the traditional O'Rourke collision scheme [1] $]$ and no-time-counter (NTC) method [19] are tested, and extended with Post and Abraham's inclusion of collision regimes [14]. For spray evaporation, Frossling's evaporation model is implemented.

\section{Summary of Outwardly-Opening Spray Modeling}

Table 1 shows a summary of various spray modeling approaches for outwardly-opening hollow-cone injectors. The modeling consists of two part; spray injection (velocity and initial SMD) and breakup. Previous modelings assumed slug flow at nozzle exit, and thus, the annular gap height and nozzle exit area are known and fixed values. Therefore, Senecal et al. []] and Das [10] calculated injection velocities from the mass flow rate and the known nozzle exit area. Pischke et al. [11] calculated the injection velocity using unsteady Bernoulli equation from pressure difference between injector and chamber, but the film thickness is assumed known. However, small gap height and varying nozzle area and shape cannot guarantee slug flows at the nozzle exit. In most experimental studies, the mass flow rate and injection pressure are known unlike varying gap height and shape. Therefore, the key feature of the present study is computing injection velocity and initial liquid film thickness using unsteady Bernoulli equation and mass conservation instead of unknown nozzle exit area and shape. In addition, the string-like structures are modeled with modified KH-RT breakup model and SMD values by imagining attached many solid-cone-like spray.

\section{Numerical Setup}

The spray characteristics of the outwardly-opening piezoelectric injector, shown in Figure 1, are investigated to find appropriate modeling of outwardly-opening hollow-cone spray for GCI engine. The experimental data as well as the operating conditions can be obtained from the internal data of Saudi Aramco and FEV. The BMW Siemens piezoelectric outwardly-opening hollow-cone GDI injector was utilized in their spray characterization. The injection pressure $\left(p_{i n j}\right)$ is $100 \mathrm{bar}$, and the chamber conditions are 5-10 bar at 300-600 $\mathrm{K}$. The actuation duration is $0.30 \mathrm{~ms}$, and the injection starts after $0.10 \mathrm{~ms}$ with the injection duration of $0.42 \mathrm{~ms}$ including opening $(0.05 \mathrm{~ms})$ and closing $(0.07 \mathrm{~ms})$ transient periods according to the experimental data. Figure 2 shows the used mass flow rate obtained from experiment, where the opening and closing ramps were assumed linear. The change in chamber pressure can be negligible because the spray is injected into constant volume chamber unlike real engine operation with compression and expansion strokes. The liquid fuel of spray is a PRF 95 mixture of 95\%-vol iso-octane and 5\%-vol $\mathrm{n}$-heptane and has a temperature of $300 \mathrm{~K}$. The closed chamber is filled with quiescent air. The injector nozzle diameter $\left(d_{\mathrm{n}}\right)$ is $4 \mathrm{~mm}$ and the maximum needle lift is $0.035 \mathrm{~mm}$.

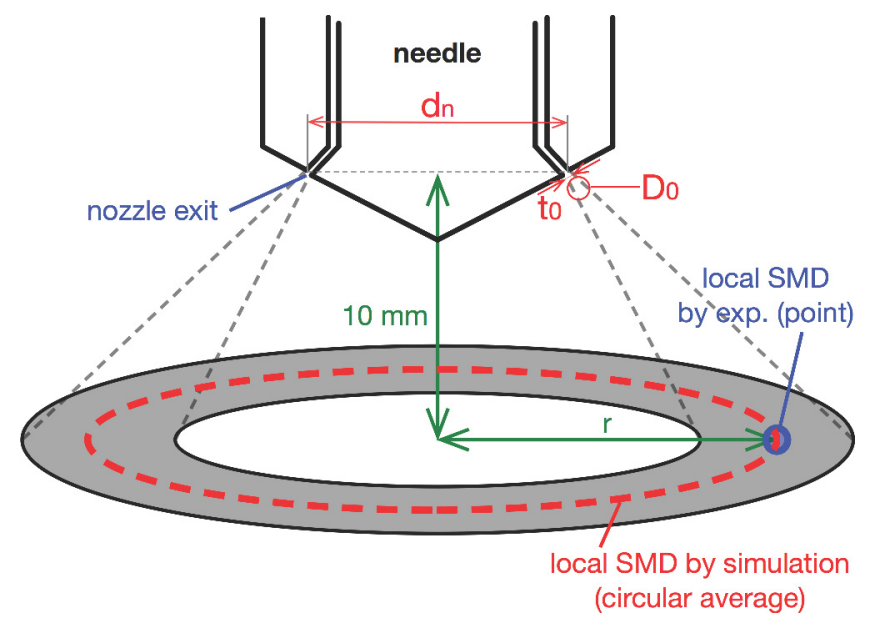

Figure 1. Schematic drawing of the outwardly-opening hollow-cone injector.

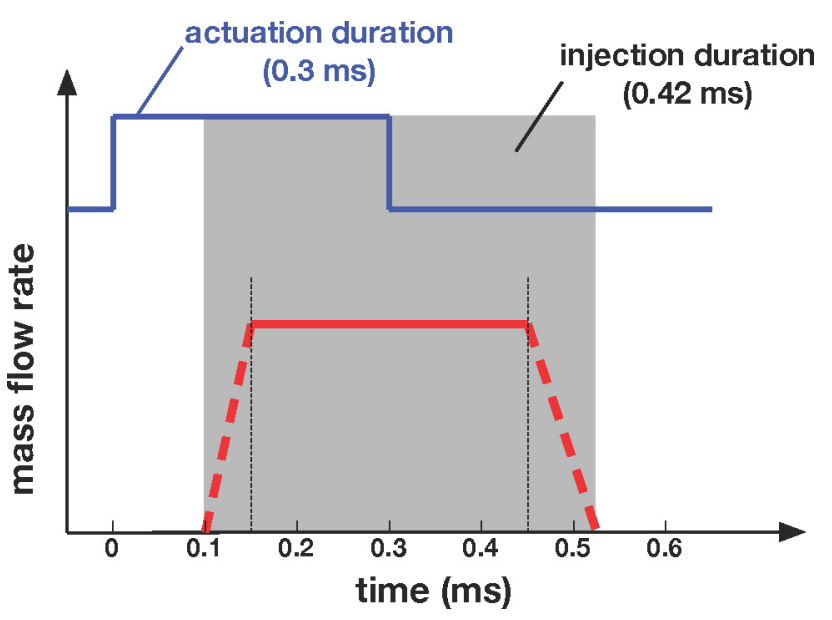

Figure 2. Injection duration and estimated mass flow rate profile.

The liquid spray is injected into the quiescent air in a cylindrical constant volume chamber with a diameter of $150 \mathrm{~mm}$ and height of $106.5 \mathrm{~mm}$ as shown in Figure 3. Adaptive mesh refinement is used for computational efficiency, and grid convergence test was conducted maintaining minimum cell size of $1 \mathrm{~mm}, 0.5 \mathrm{~mm}, 0.25$ $\mathrm{mm}$, and $0.125 \mathrm{~mm}$. In spray modeling, the grid convergence test is very challenging because collision modeling is very sensitive to number of parcels and computational time step. Figure 4 shows an example of grid convergence test of reference case 1 in Table 2 . In this test, 100,000 of parcels are injected from the nozzle and the maximum number of parcels is maintained around 0.8-1.0 millions through child parcel creations. The computational time step is fixed at $1 \mu \mathrm{s}$. Figure 4 indicates that minimum cell size should be smaller than $0.250 \mathrm{~mm}$ for grid-independent results. In subsequent study, $4 \mathrm{~mm}$ of the base mesh size is used, and the minimum cell size is maintained at $0.125-0.250 \mathrm{~mm}$ via AMR implementation. The fixed AMR embedding is implemented around nozzle exit area, and the dynamic AMR embedding is applied to flow fields based on the temperature and velocity gradients. The computational domain and the cut-plane of the refined mesh near the areas of interest are shown in Figure 3. Three cases with different chamber pressure and temperature of Table $\underline{1}$ are simulated and compared with experiments. 


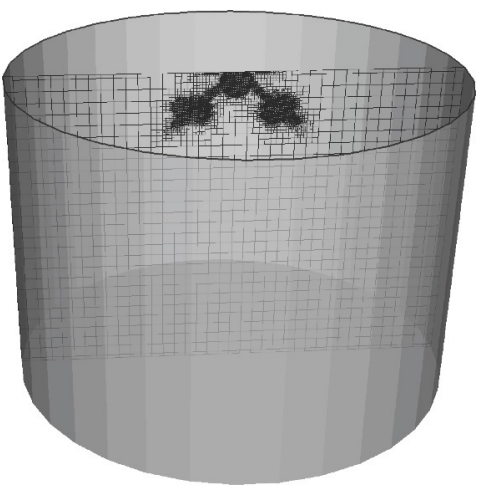

Figure 3. The computational domain of a cylindrical constant volume chamber. The adaptive mesh refinement is implemented around injector nozzle and spray regions for computational efficiency.

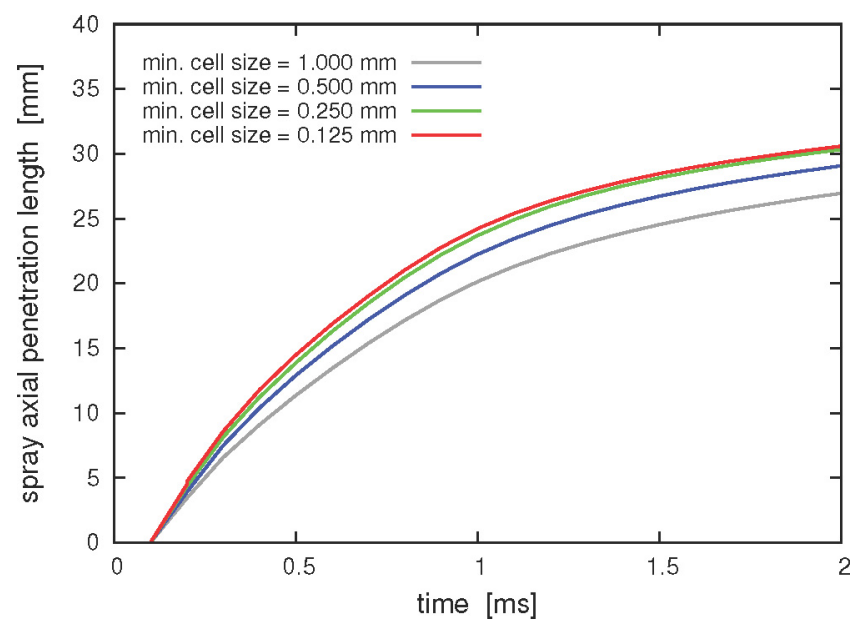

Figure 4. Grid convergence test. Adaptive mesh refinement is implemented and the minimum cell size of $1 \mathrm{~mm}, 0.5 \mathrm{~mm}, 0.25 \mathrm{~mm}$, and $0.125 \mathrm{~mm}$ are tested. The minimum cell size should be smaller than $0.25 \mathrm{~mm}$ for gridindependent simulation at the given condition.

Table 2. Simulated cases with different chamber pressure and temperature. Injection pressure and temperature is 100 bar at $300 \mathrm{~K}$ for all cases.

\begin{tabular}{|c|c|c|}
\hline & Chamber pressure, $p_{\mathrm{c}}$ & Chamber temperature, $\mathrm{T}_{\mathrm{c}}$ \\
\hline Case 1 (reference) & $5 \mathrm{bar}$ & $300 \mathrm{~K}$ \\
\hline Case 2 & $5 \mathrm{bar}$ & $600 \mathrm{~K}$ \\
\hline Case 3 & $10 \mathrm{bar}$ & $600 \mathrm{~K}$ \\
\hline
\end{tabular}

\section{Results and Discussion}

\section{Collision Models}

Before validating the new model at different conditions, collision models are first tested and compared with each other at the reference case because the widely used O'Rourke collision model [13], which is the standard approach to calculating collision probability and collision outcomes in Lagrangian spray simulations, may not be applicable for the present hollow cone spray. The traditional gasoline solid cone sprays usually have a large initial SMD, for which the breakup is a more dominant process. On the other hand, both collision and breakup models may become important in the hollowcone spray due to dense and fine spray characteristics near nozzle exit. The original O'Rourke [13] model considers only two collision
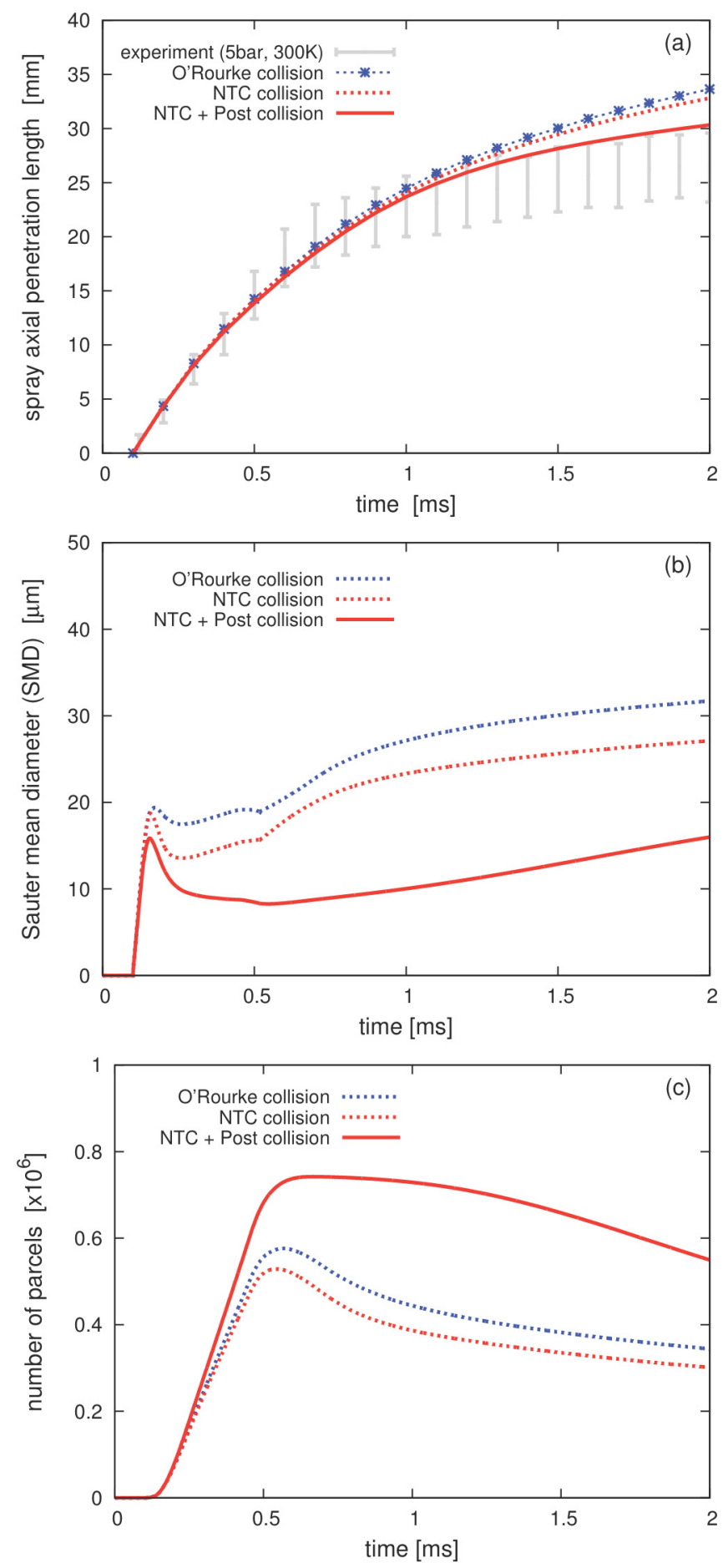

Figure 5. Comparison of collision models in the present spray modeling of outwardly opening hollow-cone spray. Reference case 1 ( 5 bar, $300 \mathrm{~K}$ ) of Table 1 is used for the test. (a) A liquid spray axial penetration length (b) SMD value histories, and (c) history of number of parcels.

regimes for simplicity; coalescence and stretching separation. Furthermore, the computational cost is proportional to the square of the number of computational parcels. Schmidt and Rutland [19] improved the computational efficiency and accuracy by proposing no-time-counter (NTC) method based on the concept of direct simulation Monte Carlo (DSMC) at the linearly-increasing computational cost with number of parcels. Post and Abraham [14] suggested a new collision outcome model for dense Diesel spray by including stretching separation and reflexive separation regimes as well as O'Rourke's two regimes. Different collision models are tested 
and compared using the present new model. Figure 5(a) shows that the collision models significantly affect the liquid penetration length because typical O'Rourke or NTC collision models causes more coalescence outcome when small droplets collide with each other in dense spray region. The larger coalescent droplets cause increased evaporating time and longer spray penetration due to a decreased liquid surface area. The occurrence of coalescence between droplets are shown clearly in Figure 5(b) and (c), where the number of parcels decreases quickly and SMD increases by overestimated coalescence in O'Rourke or NTC collision models. For computational efficiency, Post outcome model requires $30 \%$ more computational time than other models because the number of parcels increases with less coalescence rate than other models and more collision outcomes require additional computational resources. In the subsequent study, NTC collision with Post's collision outcome models is used for the observed better accuracy although it requires more computational time.

\section{Injection Distribution Models}

Three different injection distribution models were investigated and compared with each other to find the best injection distribution model before simulating cases at different conditions. It is known that Rosin-Rammler distribution produces narrower distribution than Chi-squared distribution, and represent experimental measurement better. In the hollow-cone spray, however, the initial droplet size is less than $35 \mu \mathrm{m}$, which is much smaller than typical solid-cone spray having 200-300 $\mu \mathrm{m}$ of initial droplet diameters. In these smaller initial droplet sizes, the difference of injection distribution can be negligible. In Figure 5, the narrower distribution of Rosin-Rammler, wider distribution of Chi-squared, and no-distribution of constant SMD model produces very similar spray penetration length. In this study, the constant droplet model without injection droplet variation is used in the subsequent study because we assumed the consistent liquid sheet injection from small annular gap.

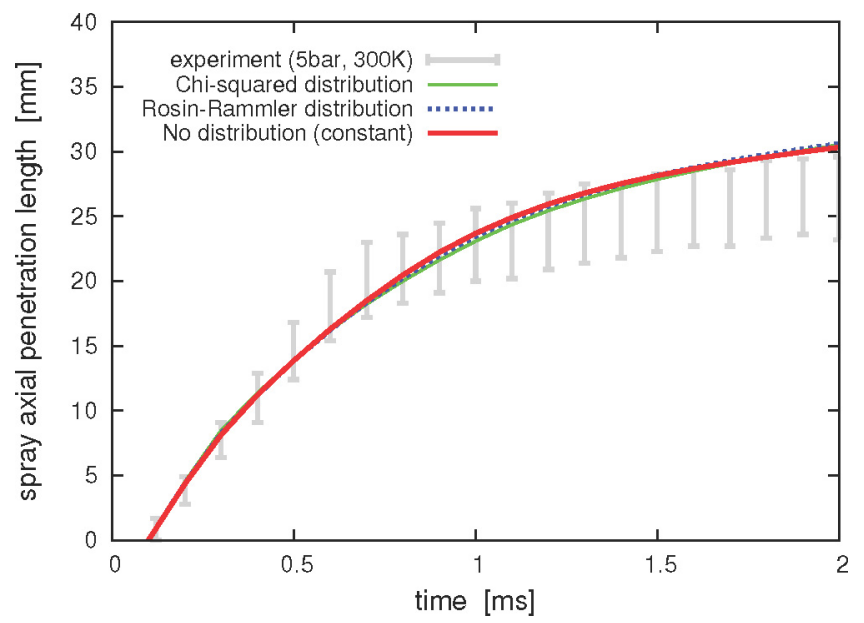

Figure 6. Comparison of injection distribution models in the present spray injection modeling.

\section{Validation of the Present Model}

For the validation of the present spray injection model, the reference case 1 of Table 1 was simulated and compared with experiment and the built-in LISA model. For the LISA breakup model, Taylor analogy breakup (TAB) is added as a secondary breakup model after the primary sheet breakup. Figure 7 shows the comparison of liquid spray axial penetration length. In the experiment, the minimum and the maximum spray tip lengths were measured while numerical simulation measures $99 \%$ of liquid mass penetration in Figure 7(a). In the beginning, both models show almost identical penetration length, and agree well with the experimental data because the spray injection by $0.52 \mathrm{~ms}$ determines the penetration length directly. However, LISA-TAB model results in shorter penetration length than the present new model after injection period. Both are located in the range of experimental spray tip measurement. However, our simulation measures $99 \%$ of liquid mass penetration, and thus, it should be closer to the higher limit of spray tip measurement in the experiment. From the definition of spray penetration, we can conclude that the new model matches better experiment than the built-in model, and our approach on the injection velocity and initial SMD are reasonable.
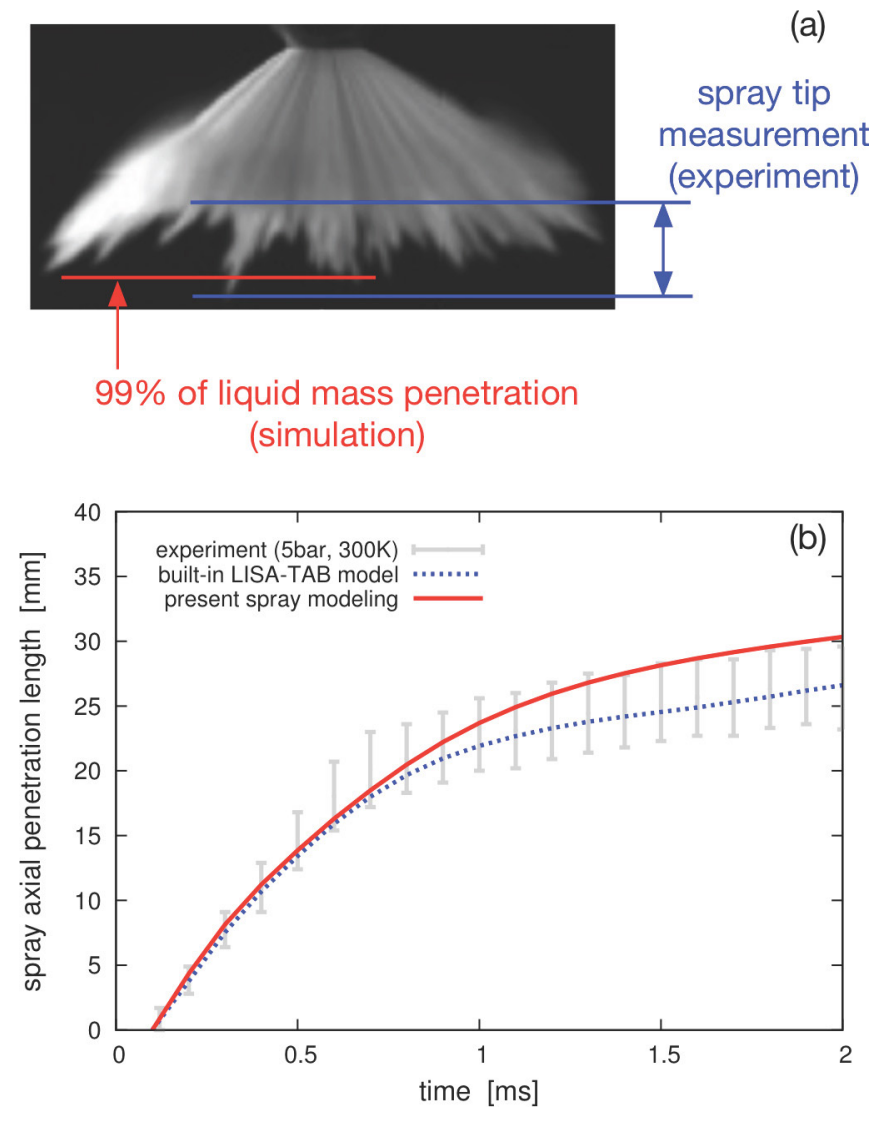

Figure 7. Comparison of spray axial penetration length between experiments and two spray injection models. (a) Definition of experimental spray tip measurement and numerical $99 \%$ of liquid mass penetration measurements. (b) Comparison of liquid spray axial penetration length between experiments, the built-in LISA-TAB model, and the present spray injection modeling.

The difference after spray injection may be caused mainly by initial drop size and following secondary breakup model. It is known that the TAB model produces unreasonably small droplets in high Weber number cases, and this may results in faster evaporation and shorter penetration length. This hypothesis can be proven clearly in the history plots of global SMD and liquid mass in Figure 8. In the built-in LISA-TAB model, the primary LISA breakup produces very small droplets less than $4 \mu \mathrm{m}$ in Figure 8(a). The global SMD history may be underestimated because the spray exists in the imaginary form of liquid sheet in the beginning and the actual size of inactive droplets cannot be accounted during primary breakup. However, the bult-in LISA model definitely produces very small droplets around a 
few micrometers. After primary breakup, the secondary breakup by TAB model usually produces unreasonably fine drops at high Weber number, and fine drops disappear faster by evaporation. Therefore, the global SMD of the built-in LISA model is small from the beginning and decreases slowly showing almost flat SMD history in Figure 8(a). The model can be improved by increasing the size constant of LISA model $\left(\mathrm{C}_{\text {lisa }}\right)$ in Table 1, but the model constant should be changed in proper range. Increasing drop size much bigger than the wavelength of maximum growth rate is not a reasonable choice. Therefore, the size constant of LISA model was set to typical value of 0.5 in this study. The liquid mass history of Figure 8(b) indicates that small size of drops by LISA-TAB model accelerate evaporation, and results in smaller liquid mass. The observed small global SMD and faster evaporation supports the shorter liquid penetration length of the built-in LISA model than the present new model.
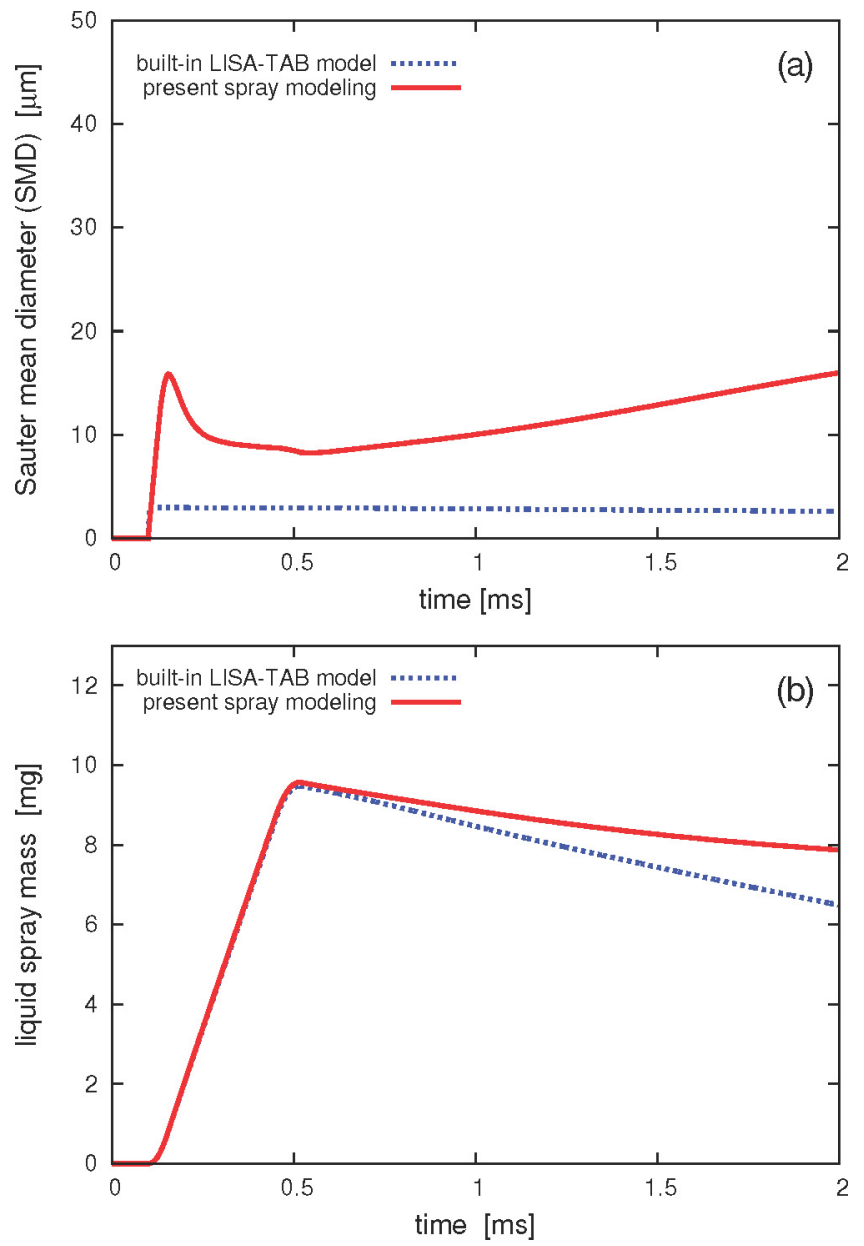

Figure 8. Comparison of global Sauter mean diameter and liquid mass between the built-in LISA-TAB model and the present new spray injection model. (a) Global Sauter mean diameter history, (b) liquid mass history.

The local SMD along radial direction at the distance of $10 \mathrm{~mm}$ from the nozzle exit was calculated and compared with experimental data in Figure 9. In experiments, the local SMD was measured at a point while this numerical study measured radial average at a given radius. The differences of measurement methods are illustrated in Figure 1. Most studies report only global SMD, and local SMD comparison is quite challenging because the modeling should simulate the details of spray structure accurately. For accurate comparison, drops smaller than $1 \mu \mathrm{m}$ were omitted when calculating local SMD because the experimental equipment cannot capture them. In the beginning, the local SMD shows peak by direct spray injection, then decreases as bigger drops breakup into smaller ones. However, the local SMD shows increase again as time goes because smaller drops evaporate or become negligibly small. The comparison with experiment shows the overall trends match well in time at different radial location of $10 \mathrm{~mm}$ and $20 \mathrm{~mm}$. Small differences may be from the breakup modeling and primarily from the variation of spray angle in the experiment. This study assumes constant spray angle during injection for easy implementation, and more studies are required to model actual experimental injection with varying spray angle and spray thickness. Another reason is the measurement approach. In the experimental study, a point measurement was used, but this study calculates the local SMD by averaging the values of annular rim as shown previously in Figure 1. Even with these differences, the comparison indicates that the present approach is able to model the detailed spray structure of the outwardly opening hollow-cone injector well.
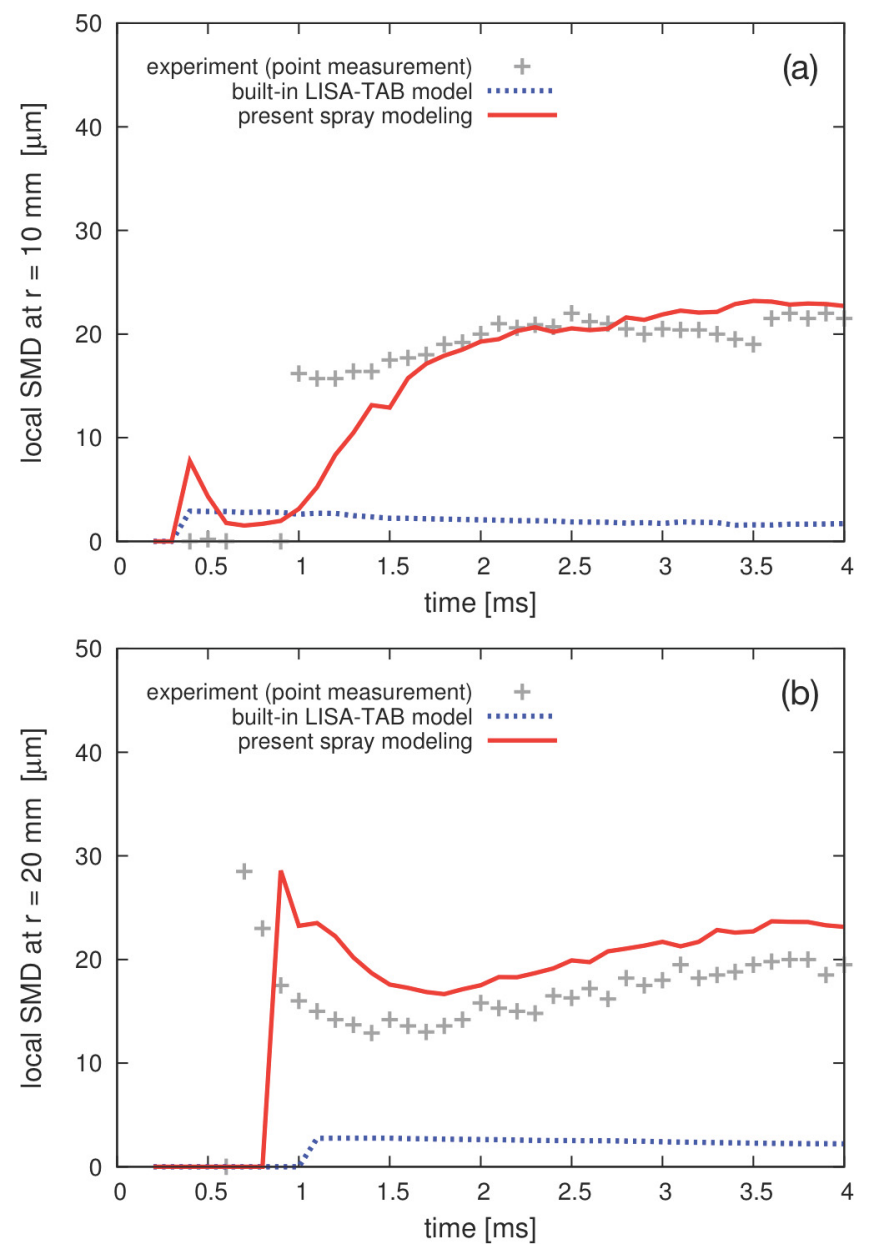

Figure 9. Local SMD measurement along radial direction at the distance of 10 $\mathrm{mm}$ from the annular nozzle exit.

It is difficult to compare computational efficiency directly between two different models because the number of parcels is different each other due to child parcel creation in the modified KH-RT model. However, the new model reports approximately $10 \%$ less computational time than the built-in LISA-TAB model under various cases with similar number of parcels. From these observations, the new model shows better accuracy with less computational time. 
The spray structure from the injector is investigated with liquid spray shapes and vorticity plots in Figure 10 . In the beginning, the spray maintains its hollow-cone shape well, but the spray starts spread out from $0.3 \mathrm{~ms}$ by vortex induced by relative velocity difference between injected spray and quiescent air. Outer vortex rings are observed clearly from 0.3 to $0.5 \mathrm{~ms}$. The spray cone spreads out more as time goes, and start to collapse from $0.8 \mathrm{~ms}$. The injection of the thin liquid sheet makes ring-like vortices and results in a big donutlike torus shape. One of the most interesting observation is that one big toroidal counter rotating vortex is divided into several pairs of counter rotating vortices at $0.8 \mathrm{~ms}$. The vortices accelerate each other, and the outer primary vortex pairs with another inner vortex of divided secondary vortices at $1.0 \mathrm{~ms}$. These multiple counter rotating vortices accelerate collapse of spray cone and spread out liquid spray more evenly.

\section{Simulations at Different Chamber Conditions}

As a further validation of the implemented spray models, two more cases with different chamber pressure and temperature in Table 1 are simulated and compared with experiments. Figure 11 shows the spray penetration length for all three cases. By the definition of numerical and experimental measurements in Figure 7, the present numerical spray penetration length should be closer to the higher limit of experimental spray tip measurement. For all cases, the present results match very well with experiments. In case 2 ( 5 bar and $600 \mathrm{~K})$, the air density is almost half of that of reference case 1 due to doubled chamber temperature. This lower air density of case 2 causes longer liquid penetration length with less drag than case 1 although evaporation occurs faster due to higher air temperature. In case 3 (10 bar and $600 \mathrm{~K}$ ), the air density is similar to the density of reference case because both chamber pressure and temperature are doubled. However, the spray injection velocity is smaller than reference case due to less pressure difference between injector and chamber. In addition, the increased breakup and evaporation due to higher pressure and temperature results in shortest spray penetration length among these three cases. The evaporation rate can be estimated in the liquid spray mass plot of Figure 12. Due to higher chamber temperature and pressure, case 3 shows fastest evaporation as expected. In case 2 , the evaporation rate is faster than reference case due to higher chamber temperature, and this may shorten the spray penetration length. In Figure 12, however, it shows longer penetration length because the decreased drop drag and following less breakup have dominant influence on the spray penetration of case 2 . All the cases including reference cases match well with experiment. The results demonstrate that the present new model can be successfully applicable for modeling of outwardly-opening hollow-cone spray, and injection velocity and initial SMD are calculated properly. For more accurate spray modeling, the injection velocity and initial liquid sheet thickness can be measured experimentally. However, measuring such case-dependent data at the nozzle exit is almost impossible due to the limitation of equipment. The new approach proposed in this study models spray injection using minimum information like mass flow rate and injection pressure, and provides good agreement with experimental data.

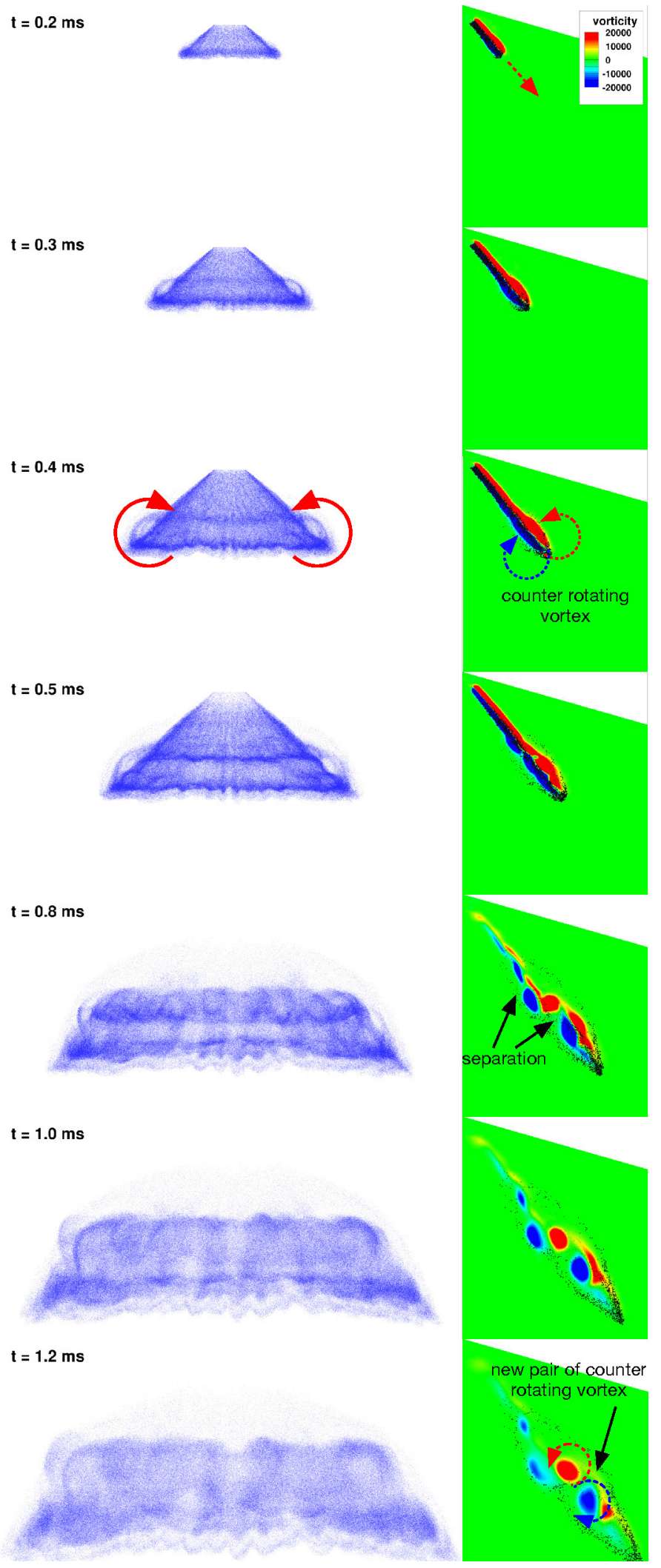

Figure 10. Snapshots of 3-D spray structures and 2-D vorticity contour on a 2-D slice through the spray. 


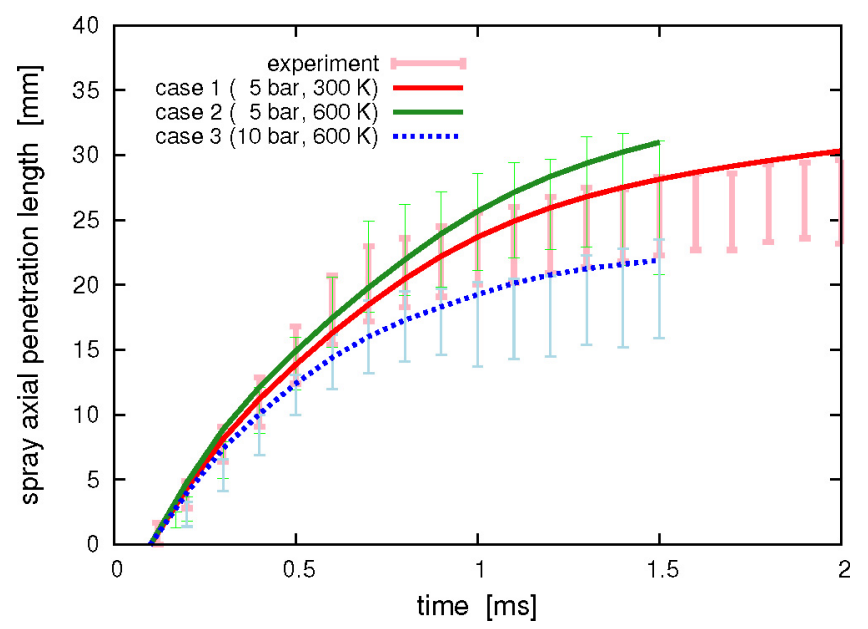

Figure 11. The comparison of spray penetration length between simulations and experiments for case 1 ( 5 bar, $300 \mathrm{~K})$, case 2 ( 5 bar, $600 \mathrm{~K})$, and case 3 (10 bar, $600 \mathrm{~K})$.

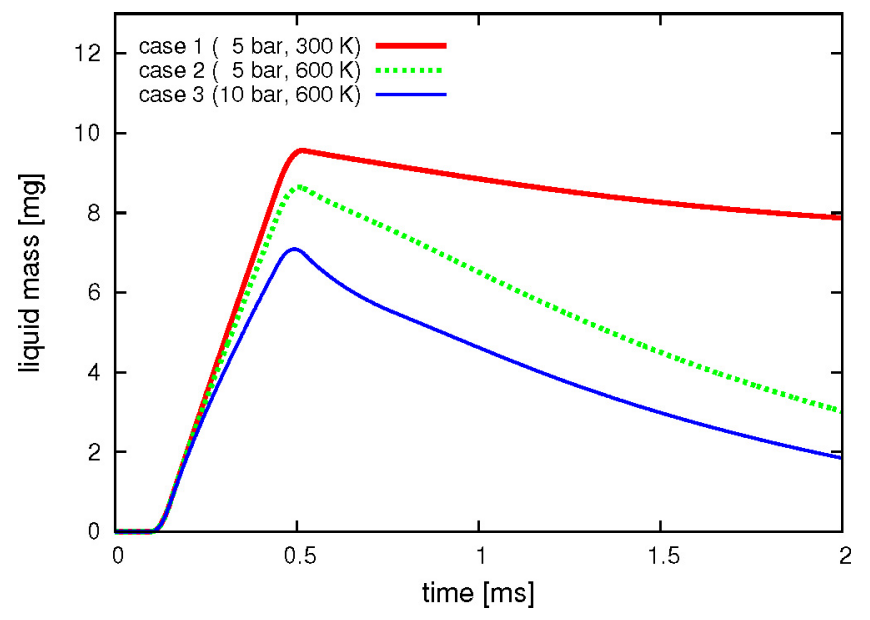

Figure 12. The history of liquid spray mass for three different cases.

\section{Conclusions}

A new spray injection model was proposed to describe the outwardlyopening piezoelectric hollow-cone injector because the traditional LISA-TAB model by toroidal ligament breakup is not suitable for representing string-like film structure observed experimentally. The injection velocity was computed from mass flow rate and pressure difference unlike slug flow assumption of previous approaches, and the initial SMD was estimated by assuming a number of attached string-shaped solid-cone injections. The spray injection was modeled using Lagrangian discrete parcel method within the framework of commercial CFD software CONVERGE, and the modified liquid injection was implemented by user-defined functions. A BMW Siemens outwardly-opening hollow-cone spray injector was characterized and validated with existing experimental data. The dense and fine spray of the injector requires more advanced collision model like Post's outcome model instead of standard O'Rourke collision model for better accuracy. The new model was found to predict the liquid penetration length and local SMD at improved fidelity compared to the built-in LISA-TAB model at various chamber pressures and temperatures.

\section{References}

1. Lefebvre, A., "Gas Turbine Combustion, Combustion: An International Series, $2^{\text {nd }}$ Edition," (Philadelphia, Taylor \& Francis, 1998), ISBN 1-56032-673-5.

2. Williams, F. A., "Combustion Theory, $2^{\text {nd }}$ Edition," (Redwood city, Addison-Wesley Publishing Company, 1985), ISBN 0-8053-9801-5.

3. Ra, Y., Loeper, P., Andrie, M., Krieger, R. et al., "Gasoline DICI Engine Operation in the LTC Regime Using TriplePulse Injection," SAE Int. J. Engines 5(3):1109-1132, 2012, doi: 10.4271/2012-01-1131.

4. Chang, J., Kalghatgi, G., Amer, A., and Viollet, Y., "Enabling High Efficiency Direct Injection Engine with Naphtha Fuel through Partially Premixed Charge Compression Ignition Combustion," SAE Technical Paper 2012-01-0677, 2012, doi: 10.4271/2012-01-0677.

5. Chang, J., Viollet, Y., Amer, A., and Kalghatgi, G., "Fuel Economy Potential of Partially Premixed Compression Ignition (PPCI) Combustion with Naphtha Fuel," SAE Technical Paper 2013-01-2701, 2013, doi:10.4271/2013-01-2701.

6. Han, Z., Parish, S., Farrell, P. V., and Reitz, R. D., "Modeling Atomization Processes of Pressure-Swirl Hollow-Cone Fuel Sprays," Atomization and Sprays 7(6):663-684, 1997, doi:10.1615/AtomizSpr.v7.i6.70.

7. Schmidt, D. P., Nouar, I., Senecal, P. K., Rutland, C. J., Martin, J. K., Reitz, R. D., and Hoffman, J. A., "Pressure-Swirl Atomization in the Near Field," SAE Technical Paper 1999-010496, 1999, doi:10.4271/1999-01-0496.

8. Senecal, P., Schmidt, D., Nouar, I., Rutland, C., Reitz, R., and Corradini, M., "Modeling High-speed Viscous Liquid Sheet Atomization," International Journal of Multiphase Flow 25:1073-1097, 1999, doi:10.1016/S0301-9322(99)00057-9.

9. Schwarz, C., Schünemann, E., Durst, B., Fischer, J., and Witt, A., "Potentials of the Spray-Guided BMW DI Combustion System," SAE Technical Paper 2006-01-1265, 2006, doi: 10.4271/2006-01-1265.

10. Das, S., "Fluid Dynamic Study of Hollow Cone Sprays," SAE Technical Paper 2008-01-0131, 2008, doi:10.4271/2008-01$\underline{0131 .}$.

11. Pischke, P., Martin, D., and Kneer, R., "Combined spray model for gasoline direct injection hollow-cone sprays," Atomization and sprays 20(4):345-364, 2010, doi:10.1615/AtomizSpr.v20. i4.60.

12. Martin, D., Cardensa, M., Pischke, P., and Kneer, R., "Experimental investigation of near nozzle spray structure and velocity for a GDI hollow cone spray," Atomization and Sprays 20(12):1065-1076, 2010, doi:10.1615/AtomizSpr.v20.i12.40.

13. O'Rourke, P., “Collective Drop Effects on Vaporizing Liquid Sprays," Ph.D. thesis, Princeton University, 1981.

14. Post, S. L. and Abraham, J., "Modeling the outcome of dropdrop collisions in Diesel sprays," International Journal of Multiphase Flow 28(6):997-1019, 2002, doi:10.1016/S03019322(02)00007-1. 
15. Munnannur, A., and Reitz, R., "A new predictive model for fragmenting and non-fragmenting binary droplet collisions," International Journal of Multiphase Flow 33(8):873-896, 2007, doi:10.1016/j.ijmultiphaseflow.2007.03.003.

16. Senecal, P., Richards, K. and Pomraning, E., "CONVERGE (version 2.2.0) Manual", (Madison, WI, Convergent Science Inc., 2014).

17. Senecal, P. K., Richards, K. J., Pomraning, E., Yang, T., Dai, M. Z., McDavid, R. M., Patterson, M. A., Hou, S., and Shethaji, T., "A New Parallel Cut-Cell Cartesian CFD Code for Rapid Grid Generation Applied to In-Cylinder Diesel Engine Simulations," SAE Technical Paper 2007-01-0159, 2007, doi:10.4271/200701-0159.

18. Badra, J. A., Sim, J., Elwardany, A., Jaasim, M., et al., "Numerical Simulations of Hollow Cone Injection and Gasoline Compression Ignition Combustion with Naphtha Fuels", Journal of Energy Resources Technology, In press, JERT-16-1011, 2016.

19. Schmidt, D. P. and Rutland, C., "A New Droplet Collision Algorithm," Journal of Computational Physics 164(1):62-80, 2000, doi:doi:10.1006/jeph.2000.6568.

\section{Contact Information}

\section{Dr. Jaeheon Sim}

Clean Combustion Research Center

King Abdullah University of Science and Technology

Saudi Arabia

+966 (12) 808-4758

Jaeheon.Sim@,kaust.edu.sa

\section{Acknowledgments}

This work was sponsored by the Fuel Technology Division at Saudi Aramco R\&DC. The work at King Abdullah University of Science and Technology (KAUST) was funded by KAUST and Saudi Aramco under the FUELCOM program. We also acknowledge the help and support from Convergent Science Inc. (CSI).

\section{Definitions/Abbreviations}

AMR - adaptive mesh refinement

CFD - computational fluid dynamics

GCI - gasoline compression ignition

GDI - gasoline direct injection

HCCI - homogeneous charge compression ignition

KH-RT - Kelvin-Helmholtz and Rayleigh-Taylor

LISA - linear instability sheet atomization

NTC - no-time-counter
RANS - Reynolds-averaged Navier-Stokes

RNG - Reynolds normalization group

SMD - Sauter mean diameter

TAB - Taylor analogy breakup

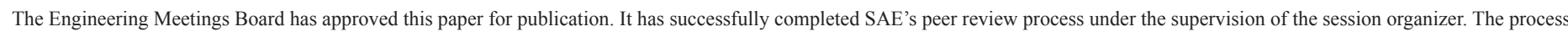
requires a minimum of three (3) reviews by industry experts.

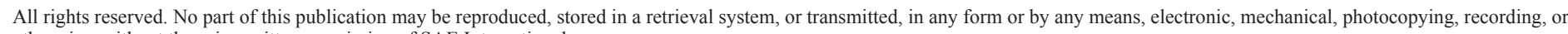
otherwise, without the prior written permission of SAE International.

Positions and opinions advanced in this paper are those of the author(s) and not necessarily those of SAE International. The author is solely responsible for the content of the paper.

ISSN 0148-7191

http://papers.sae.org/2016-01-0844 